\title{
Kergin 插值算子的扩张
}

\author{
贾荣庆 \\ (得江大学数学系, 杭州)
}

在 1978 年，Kergin ${ }^{\text {[1] }}$ (又见文献[2])引人了一种对多变量光滑函数插值的新方法. 这一 方法是 Lagrange 插值的一种自然推广. 现在这种插值称做 Kergin 插值.

我们先来引进一些记号. 令 $\mathbb{R}^{k}$ 是 $k$ 维实向量空间. 对于 $x \in \mathbf{R}^{k}$, 我们用 $x_{i}$ 表示 $x$ 的第 $i$ 个分量. 对于两个向量 $x$ 及 $y$, 其内积记为 $x \cdot y:=\Sigma x_{i} y_{i}$. 令 $e^{i}$ 为第 $i$ 个分量为 1 其余分 量为 0 的向量. 对任意 $y \in \mathbb{R}^{k} \backslash\{o\}$, 函数 $f$ 的方向导数 $D_{y} f$ 定义为

$$
D_{y} f:=\lim _{t \rightarrow 0}(f-f(\cdot-t y)) / t .
$$

特别, $D_{e^{i}}$ 将简写为 $D_{i}$. 我们将利用通常的多重指标 (multi-index) 记号. 例如

$$
D^{\alpha}:=D_{1}^{a_{1}} \cdots D_{k}^{a_{k}} \text {, 对于 } \alpha=\left(\alpha_{1}, \cdots, \alpha_{k}\right) \in \mathbf{Z}_{+}^{k} \text {. }
$$

令 $C^{(n)}\left(\mathbf{R}^{k}\right)$ 表示所有 $n$ 阶光滑的函数 $f: \mathbf{R}^{k} \rightarrow \mathbf{R}$ 组成的线性空间. 对于 $\mathbb{R}^{k}$ 的一个子集 $K$, 范数 $\|\cdot\|_{n, \mathrm{~K}}$ 定义为

$$
C^{(n)}(K):=\left\{\left.f\right|_{K} ; f \in C^{(n)}\left(\mathbb{R}^{k}\right)\right\} \text {. }
$$

$$
\|f\|_{n, K}=\sum_{|a| \leqslant n}\left\|D^{\alpha} f\right\|_{\infty, K} .
$$

如果从上下文, $K$ 的意义是清楚的, 下标 “ $K$ ” 将省去. 如果不作相反声明, $C^{(n)}(K)$ 将赋以范 数 $\|\cdot\|_{n, K}$. 我们以 $\pi_{m}\left(\mathbb{R}^{k}\right)$ 表示所有全次数 $\leqslant m$ 的 $k$ 元多项式所成的空间. 对于 $g \epsilon$ $\pi_{m}\left(\mathbf{R}^{k}\right), g(x)=\sum_{|a| \leqslant m} C_{\alpha} x^{\alpha}, q(D)$ 定义为 $\sum_{|a| \leqslant m} C_{\alpha} D^{\alpha}$. 一个集合 $E$ 的基数记为 Card $E$.

现在我们可以叙述 Kergin 的结果.

定理 $\mathbf{K}$ (见 Kergin ${ }^{\mathfrak{a}, 2]}$ ) 给定 $n+1$ 个点 $x^{0}, \cdots, x^{n} \in \mathbf{R}^{k}$ ，存在一个唯一的线性映 射 $P: C^{(n)}\left(\mathbf{R}^{k}\right) \rightarrow \pi_{s}\left(\mathbf{R}^{k}\right)$ 具有下述性质: 对于每一个 $f \in C^{(n)}\left(\mathbb{R}^{k}\right)$, 每一个 $q \in \pi_{l}\left(\mathbb{R}^{k}\right)$ 及每 一个子集 $J \subset\{0,1, \cdots, n\}, \operatorname{Card} J-l+1(l \leqslant n)$, 在集 $\left\{x^{j} ; j \in J\right\}$ 的凸包里必然存在一 个点 $x$ 使得

$$
q(D)(P f)(x)=q(D) f(x) .
$$

稍后, Micchelli 与 $\mathrm{Milman}^{[3]}$ (又见 Micchelli ${ }^{[4]}$ ) 给出了 Kergin 插值多项式的显式表 示:

$$
P f(x)=\int_{\left[x^{0}\right]} f+\int_{\left[x^{0}, x^{1}\right]} D_{x-x^{0}} f+\cdots+\int_{\left[x^{0}, x^{1}, \cdots, x^{n}\right]} D_{x-x 0} \cdots D_{x-x^{n-1}} f,
$$

其中

$$
\int_{\left[x^{0}, x^{1}, \cdots, x^{n}\right]} f:=\int_{S^{n}} f\left(x^{0}+u_{1}\left(x^{1}-x^{0}\right)+\cdots+u_{n}\left(x^{n}-x^{0}\right)\right) d u,
$$

本文 1985 年 7 月 12 日收到。 
而 $S^{n}$ 是 $\mathbb{R}^{n}$ 里的标准单纯形:

$$
S^{n}=\left\{u=\left(u_{1}, \cdots, u_{n}\right) ; u_{i} \geqslant 0, \Sigma u_{t} \leqslant 1\right\} .
$$

设 $K$ 是一个包含 $x^{0}, \cdots, x^{n}$ 的紧凸集. 由上述 $P f$ 的表示我们看出 $P$ 是从 $C^{(n)}(K)$ 到 $\pi_{n}(K)$ 的一个有界算子. 与一元的情形不同, 即使插值点两两不同, Kergin 插值也需要知道被插值 函数的直至 $n$ 阶的导数. 这样, 考虑如下的问题是有意义的：是否有可能将 Kergin 插值算 子推广为 $C^{(l)}(K)$ (某个 $l<n$ ) 上的有界算子?

在这方面, Kergin 证明了当 $k<n$ 且 $x^{0}, \cdots, x^{n}$ 占有最广位置时, 即 $\left\{x^{0}, \cdots, x^{n}\right\}$ 中任 意 $k+1$ 个点形成一个 $k$ 维的单纯形, 那么 $P$ 是 $C^{k-1}\left(\mathbf{R}^{k}\right)$ 上的有界算子. Micchelli ${ }^{[4]}$ 推 广了 Kergin 的结果,他得到了

定理 $\mathbf{M}$ 线性泛函

$$
f \mapsto \int_{\left[x^{0}, \ldots, x^{n}\right]} D_{y} f
$$

可以扩张为 $C(K)$ 上的有界泛函的充分必要条件是 $y$ 落在由 $x^{1}-x^{0}, \cdots, x^{n}-x^{0}$ 张成的 线性空间内.

对于一般情形, Micchelli 指出, 如果 $g=\sum_{j=0}^{m} q_{i}$, 其中 $q_{i}$ 是次数为 $j$ 的齐次多项式,且若

$$
T: f \mapsto \int_{\left[x^{0}, \ldots, x^{n}\right]} q(D) f, f \in C^{(n)}(K),
$$

具有到 $C^{(l)}(K)$ 上的有界扩张, 那么由 $\lambda \perp x^{j}-x^{0}, j=1, \cdots, n$ 可以推得 $q_{l+1}(\lambda)=\cdots=$ $q_{m}(\lambda)=0$. 在同一篇文章里, Micchelli 提出如下的问题: 上述条件对于 $T$ 在 $C^{(l)}(K)$ 上的 有界性是否是充分的?

本文的目的是要回答上述问题。首先,我们注意到上述条件对于 $T$ 在 $C^{(l)}(K)$ 上的有界 性不是充分的. 一个反例: $k=2, l=0, n=1, x^{0}=(0,0), x^{1}=(1,0), q(x)=x_{1}^{2}$. 在下 面, 我们将给出一个对于 $T$ 在 $C^{(l)}(K)$ 上为有界的充分且必要的条件. 为此, 我们要引人极 大重数的概念. 一个序列 $\left(y^{i}\right)_{0}^{n}$ 的极大重数定义为 $\max \left\{r ;\left(y^{i}\right)_{0}^{n}\right.$ 里有 $r$ 个元素重合 $\}$.

定理 1 给定 $x^{0}, \cdots, x^{n} \in \mathbf{R}^{k}$, 及 $q=\sum_{j=0}^{m} q_{i}$, 对每一个 $i, q_{i}$ 是次数为 $i$ 的齐次多项 式. 对任意 $\lambda \in \mathbf{R}^{k}, r(\lambda)$ 表示序列 $\left(\lambda \cdot x^{i}\right)_{i=0}^{n}$ 的极大重数. 那么由(2)式给出的线性泛函 $T$ 具有到 $C^{(l)}(K)(0 \leqslant l<n)$ 上的有界扩张的充分必要条件是

对所有 $\lambda \in \mathbb{R}^{k} \backslash\{0\}$ 及 $i>n+1-r(\lambda)+l, q_{i}(\lambda)=0$.

证设 $\left(t_{i}\right)_{0}^{n}$ 是实数的一个非减序列. 置

$$
s_{i}:=\max \left\{s ; t_{i}=t_{i-s}\right\} \text {. }
$$

如同往常, $M\left(\cdot \mid t_{0}, \cdots, t_{n}\right)$ 表示在节点序列 $\left(t_{i}\right)_{0}^{n}$ 上的规范 $n$ 阶 $B$-样条:

$$
M\left(t \mid t_{0}, \cdots, t_{n}\right):=n\left[t_{0}, \cdots, t_{n}\right](\cdot-t)_{+}^{n-1} \text {. }
$$

我们用 $\delta_{t}$ 表示由 $\delta_{t} f=f(t)$ 所决定的广义函数. 在以下的证明中，我们要用到如下的引 理,其证明可由文献 [5]的第二章及第四章的有关结果导出.

引理 对任意非负整数 $i$,

$$
D^{i} M\left(\cdot \mid t_{0}, \cdots, t_{n}\right)=\sum_{i+s_{i} \geqslant n} C_{i j} D^{i+s_{i}-n} \delta_{t_{i}}+\text { 一个 } L_{\infty} \text {-函数, }
$$

其中 $c_{i i}$ 为实数, 且 


$$
c_{i j} \neq 0 \text { 当 } t_{i}<t_{i+1} \text {. 时. }
$$

我们先就 $k=1$ 这一情形证明定理 1. 假定

$$
q(t)=\sum_{j=0}^{m} a_{j} t^{i}, a_{m} \neq 0 .
$$

为方便起见, 记 $t_{i}=x^{i}, i=0, \cdots, n$ 。 令 $d:=\max _{i}\left\{s_{i}+1\right\}$, 其中 $s_{i}$ 是由(4)式给出的. 如 果条件 (3)成立,亦即 $m \leqslant n+1-d+l$, 那么对任意 $i, m-l \leqslant n-s_{i}$. 从文献 [4]我们 有

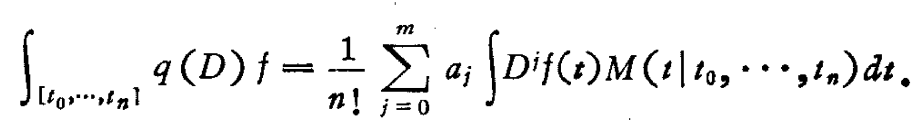

当 $j \leqslant l$ 时,显然

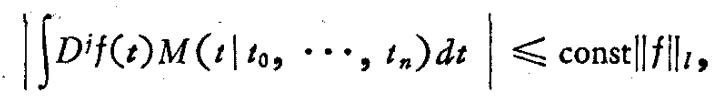

其中 const 表示一个不依赖于 $f$ 的常数. 当 $j>l$ 时, 我们应用引理的结果. 由于对任意 $i$, $m-l+s_{i}-n \leqslant 0$, 我们有

由分部积分法,我们得到

$$
D^{i-l} M\left(\cdot \mid t_{0}, \cdots, t_{n} j=\sum_{j-l+s_{i}=n} c_{i j} \delta_{t_{i}}+\text { 一个 } L_{\infty}\right. \text {-函数. }
$$

$$
\int D^{i f}(t) M\left(t \mid t_{0}, \cdots, t_{n}\right) d t=(-1)^{i-l} \int D^{l} f(t) D^{i-l} M\left(t \mid t_{0}, \cdots, t_{n}\right) d t .
$$

所以对于 $i>l$, (5)式也是成立的. 这样, 条件(3)的充分性已经得到了证明. 现要证明条 件(3)的必要性. 如果条件(3)不成立, 那么 $m>n+1-d+l$, 因此对某个 $i, t_{i}<t_{i+1}$ 且 $m+s_{i}-n>l$. 对任意实数 $A$, 我们可以找到 $f \in C^{(n)}(K)$ 使得 $\|f\|_{l}=1, f^{\left(m+s_{p}-n\right)}\left(t_{p}\right)=0$ 对于 $p \neq i$ 且 $f^{\left(m+s_{i}-n\right)}\left(i_{i}\right)=A$. 这样,

$$
\begin{aligned}
& \int_{\left[t_{0}, \cdots, t_{n}\right]} q(D) f=\frac{1}{n !} \sum_{j=0}^{m}(-1)^{i} \int f(t) D^{i} M\left(t \mid t_{0}, \cdots, t_{n}\right) d t \\
& =(-1)^{n-s_{i}} c_{i m} a_{m} f^{\left(m+s_{i}-n\right)}\left(t_{i}\right) / n !=(-1)^{n-s_{i}} c_{i m} a_{m} A / n ! .
\end{aligned}
$$

由于 $A$ 可以是任意实数, 而 $c_{i m} a_{m} \neq 0$, 由此 $T$ 在 $\left(C^{(n)}(K),\|\cdot\|_{l}\right)$ 上是无界的, 即 $T$ 不能 扩张为 $C^{(2)}(K)$ 上的有界泛函. 这就证明了条件(3)的必要性.

对于一般的 $k$, 我们将利用提升 (lifting) 这一技巧. 考虑形如 $f=g \circ \lambda$ 的函数, 其中 $g \in C^{(n)}\left(\mathbb{R}^{1}\right)$ 且 $\lambda \in \mathbb{R}^{k}$. 已知集合

$$
E=\operatorname{span}\left\{\left.g \circ \lambda\right|_{K} ; g \in C^{(n)}\left(\mathbb{R}^{1}\right), \lambda \in \mathbb{R}^{k},\|\lambda\|_{\infty}=1\right\}
$$

在空间 $C^{(l)}(K)$ 中稠密, $0 \leqslant l \leqslant n$. 这样, $T$ 可以有界地扩张到 $C^{(l)}(K)$ 上的充分必要条件 是 $T$ 在 $\left(E,\|\cdot\|_{l}\right)$ 上为有界. 已知(见文献[4])对于 $f=g \circ \lambda$,

$$
\int_{\left[x^{0}, \ldots, x^{n}\right]} q(D) f=\sum_{j=0}^{m} q_{i}(\lambda) \int_{\left[\lambda \cdot x^{0}, \ldots, \lambda \cdot x^{n}\right]} D^{i f} .
$$

由已经证明的事实可知, $T$ 在 $C^{(l)}(K)$ 上为有界的充分必要条件是(3)成立. 这就完成了定理 1 的证明.

上述方法也可用于研究 Kergin 插值算子的扩张问题. 我们有

定理 2 由 (1) 式定义的 Kergin 插值算子 $P$ 具有到 $C^{(l)}(K)$ 上的有界扩张的充分必 
要条件是 $\left\{x^{0}, \cdots, x^{n}\right\}$ 之中的任意 $l+2$ 个点张成 $\mathbf{R}^{k}$.

证 对任意 $\lambda \in \mathbf{R}^{k}$, 记 $K_{\lambda}=\lambda \cdot K=\{\lambda \cdot x ; x \in K\}$. 对任意 $g \in C^{(n)}\left(\mathbf{R}^{1}\right)$, 令 $P_{\lambda} g$ 是在点 $\lambda \cdot x^{0}, \cdots, \lambda \cdot x^{n}$ 处对 $g$ 插值的 $n$ 次多项式, 那么 $P_{2}$ 是 $C^{(n)}\left(K_{\lambda}\right)$ 上的线性算子. 已 知(见文献 [6]) Kergin 插值算子 $P$ 满足

$$
P(g \circ \lambda)=P_{\lambda} g \circ \lambda \text {. }
$$

所以 $P$ 在 $\left(E,\|\cdot\|_{l}\right)$ 上为有界当且仅当对于每个 $\lambda \in \mathbf{R}^{k},\|\lambda\|_{\infty}=1, P_{2}$ 在 $C^{(l)}\left(K_{2}\right)$ 上为有界.

先证充分性. 由于 $\left\{x^{0}, \cdots, x^{n}\right\}$ 之中任意 $l+2$ 个点张成 $\mathbb{R}^{k}$, 序列 $\left(\lambda \cdot x^{i}\right)_{0}^{n}$ 的极大重 数不超过 $l+1$. 由差商公式 (见文献 [5] 公式 (2.89)) 及多项式插值的牛顿公式我们断言 $P_{\lambda}$ 在 $C^{(l)}\left(K_{\lambda}\right)$ 上是有界的.

现假定 $\left\{x^{0}, \cdots, x^{n}\right\}$ 之中有 $l+2$ 个点, 它们不张成 $\mathbf{R}^{k}$. 那么存在 $\lambda \in \mathbf{R}^{k},\|\lambda\|_{\infty}=1$, 使得序列 $\left(\lambda \cdot x^{i}\right)_{i=0}^{n}$ 的极大重数至少为 $l+2$. 记 $t_{i}=\lambda \cdot x^{i}$, 不失普遍性, 我们可以假定 $\left(t_{i}\right)_{0}^{n}$ 是一个非减的实数序列. 令 $s_{i}$ 如同 (4)式所定义的. 那么必存在某个 $i$ 使得 $s_{i}>l+$ 1. 对任意实数 $A$, 我们可以找到 $g \in C^{(n)}\left(K_{2}\right)$ 使得 $\|g\|_{l}=1, g^{\left(s_{p}\right)}\left(t_{p}\right)=0$ 对于 $p \neq i$ 且 $g^{\left(s_{i}\right)}\left(t_{i}\right)=A$. 此时我们有

$$
P_{\lambda} g(t)=A \frac{\left(t-t_{i}\right)^{s_{i}}}{s_{i} !} \prod_{t_{j} \neq t_{i}} \frac{t-t_{i}}{t_{i}-t_{j}} .
$$

由于 $A$ 可以为任意实数, $P_{2}$ 在 $C^{(l)}\left(K_{\lambda}\right)$ 上是无界的. 这就完成了定理 2 的证明.

\section{參文献}

[ 1] Kergin, P., Interpolation of $\mathrm{C}^{k}$ functions, $\mathrm{Ph} . \mathrm{D}$. Thesis, U. Toronto, Toronto, Canada, 1978.

[ 2 ] Kergin, P., A natural interpolation of $C^{k}$ functions, J. Approx. Theory, 29 (1980), 278-293.

[ 3 ] Micchelli, C A. and Milman, P., A formula for Kergin interpolation in $\mathrm{R}^{k}$, J. Approx. Theory, 29 (1980), 294-296.

[ 4] Micchelli, C. A., A constructive approach to Kergin interpolation in $R^{k}$ : Multivariate B-splines and Lagrange interpolation, Rocky Moutain J. Math., 10(1980), 485-497.

[ 5 ] Schumaker, L. L., Spline Functions: Basic Theory, John Wiley and Sons, New York, 1981.

[6] de Boor, C., Polynomial interpolation, Proc. Int. Cong. Helsinki, 1978, 917-922. 